7th International Workshop on Astronomy and

Relativistic Astrophysics (IWARA 2016)

International Journal of Modern Physics: Conference Series

Vol. 45 (2017) 1760036 (4 pages)

(C) The Author(s)

DOI: $10.1142 / \mathrm{S} 2010194517600369$

\title{
A Model for the Braking Indices of Pulsars
}

\author{
Heitor O. de Oliveira, Rubens M. Marinho Jr. \\ Departamento de Física \\ Instituto Tecnológico de Aeronáutica (ITA) \\ Praca Marechal Eduardo Gomes 50, 12228-900 São Jose dos Campos, SP, Brazil \\ heitoroliveiradeoliveira@gmail.com,marinho.rubens@gmail.com \\ Nadja S. Magalhães \\ Departamento de Física \\ Universidade Federal de São Paulo (UNIFESP) \\ Rua São Nicolau 210, 09913-030 Diadema, SP, Brazil \\ nadjasm@gmail.com
}

Published 15 August 2017

\begin{abstract}
Stars known as pulsars are generally modeled as magnetized spheres made of neutrons with high rotation frequency. It is known that such stars are spinning down and this braking is measured by a parameter, n, known as braking index. For the canonical model such parameter should have a single value for all pulsars: $\mathrm{n}=3$. However, from observations it is known that $\mathrm{n}$ diverges from 3 . In this work, differently from the canonical model, we have hypothesized the existence of a variation of the moment of inertia of the star through a time-varying radius. Using energy conservation we find the values for the variation of the radius of our pulsar sample. Our results indicate that it may be reasonable to consider that the radius of pulsars can be changing with time.
\end{abstract}

Keywords: Pulsars, Star Rotation, Magnetic Fields.

PACS numbers: 97.60.Jd, 97.60.Gb, 26.60

\section{Introduction}

Pulsars are generally modeled as neutron stars, and they are referred to as pulsating because signals received from them arrive in the form of well-defined radio pulses ${ }^{1}$. This effect is basically explained assuming a strong magnetic field and high speed rotation of a neutron star, leading to radiation in the form of a rotating beacon ${ }^{2}$ whose emission is given by a rotating dipole with magnetic axis inclined relative to the axis of rotation ${ }^{3}$.

This is an Open Access article published by World Scientific Publishing Company. It is distributed under the terms of the Creative Commons Attribution 4.0 (CC-BY) License. Further distribution of this work is permitted, provided the original work is properly cited. 
Traditionally the canonical model for pulsars is accepted to explain the pulses and it predicts a slow-down quantified by a parameter $n$ (known as braking index) equal to 3. However, for pulsars with braking index calculated from observations (B0531+21, B0833-45, B0540-69, B1509-58, J1846-0258, J1119-6127 and J17343333 ) the values of $n$ are different and less than 3, as shown in Table 1. The complexity in understanding the phenomenon that leads to $n \neq 3$ has been investigated by many authors ${ }^{4-11}$ and is still an open problem. In this work we summarize our proposed modification in the canonical model, where we consider a uniform variation in a pulsar's radius to explain the different values for $n$.

\section{The Canonical Model for Pulsars' Spin-Down}

The model called canonical assumes that a pulsar is radiating its rotational energy $\left(E_{\text {rot }}\right)$ solely through its magnetic dipole $(m)$, i.e., in the form of electromagnetic energy $\left(E_{m r}\right)$ and therefore the variation of its energy is given as follows:

$$
\dot{E}_{r o t}+\dot{E}_{m r}=0
$$

A magnetic dipole misaligned relative to its axis of rotation radiates energy $\operatorname{as}^{12}$ :

$$
\dot{E}_{m r}=\frac{2}{3 c^{3}}|\ddot{m}|^{2}
$$

where $c$ is the speed of light in vacuum and $m$ is the magnetic dipole moment that in vector form is:

$$
\vec{m}=\frac{B_{P} R^{3}}{2}(\cos \alpha \hat{k}+\sin \alpha \cos (\Omega t) \hat{i}+\sin \alpha \sin (\Omega t) \hat{j})
$$

In this canonical model, this equation has the following constants: $B_{P}$ is the magnetic field at the pole, $R$ is the radius of the pulsar and $\alpha$ is the angle between the axis of the magnetic dipole and the pulsar rotation axis, while $\Omega$ is the pulsar's rotation speed, which varies with time. Then the magnetic dipole power becomes:

$$
\dot{E}_{m r}=\frac{1}{6 c^{3}} B_{P}^{2} R^{6} \Omega^{4} \sin ^{2} \alpha=k I \Omega^{4}
$$

For the rotational energy of a pulsed star we have

$$
E_{\text {rot }}=\frac{I \Omega^{2}}{2}
$$

where $I$ is the moment of inertia that in the canonical model is assumed to be constant $(\dot{I}=0)$. Consequently, the variation of this rotational energy (or rotation power) is given by

$$
\dot{E}_{\text {rot }}=I \cdot \Omega \cdot \dot{\Omega}
$$

where the dot denotes a time derivative. 
From the energy variations in Eq. (4) and Eq. (6) the Eq. (1) results in:

$$
\dot{\Omega}=-k \Omega^{3} \text {, }
$$

where $\mathrm{k}$ is a positive constant.Therefore, the canonical model predicts a gradual decay of the rotation frequency.

A pulsar's spin-down can be quantified by a parameter known as braking index $(n)$, given by the observables $\Omega, \dot{\Omega}$ and $\ddot{\Omega}$ according to the definition

$$
n \equiv \frac{\Omega \ddot{\Omega}}{\dot{\Omega}^{2}} .
$$

For the canonical model Eq. (7) yields $n=3$.

\section{Modifying the Canonical Model}

We propose a uniform time variation in the radius of pulsars. As a consequence, the mathematical expressions of the canonical model change yielding

$$
n=\frac{3 R \dot{\Omega}+3 \dot{R} \Omega}{R \dot{\Omega}-\dot{R} \Omega} .
$$

In Table 2 we present the results from Eq. (9) assuming the existence of a uniform variation of the radius for our sample of pulsars, whose $n$ values are reported in Table 1 .

Table 1. Angular velocity $(\dot{\Omega})$ and its first time derivatives for the sample of pulsars.

\begin{tabular}{ccccc}
\hline Pulsar & $\begin{array}{c}\Omega \\
\left(\mathrm{rad} \mathrm{s}^{-1}\right)\end{array}$ & $\begin{array}{c}\dot{\Omega} \\
\left(\times 10^{-10} \mathrm{rad} \mathrm{s}^{-2}\right)\end{array}$ & $\begin{array}{c}\ddot{\Omega} \\
\left(\times 10^{-21} \mathrm{rad} \mathrm{s}^{-3}\right)\end{array}$ & $\mathrm{n}$ \\
\hline B 0531+21 & $189.912022^{13}$ & $-24.2674^{13}$ & $78.075^{13}$ & $2.51^{18}$ \\
B 0833-45 & $70.4^{14}$ & $-0.986^{14}$ & $0.19^{14}$ & $1.4^{18}$ \\
B 0540-69 & $124.623817^{15}$ & $-11.8365^{15}$ & $25.6^{15}$ & $2.28^{18}$ \\
B 1509-58 & $41.68013054^{16}$ & $-4.24618765^{16}$ & $12.2944^{16}$ & $2.839^{18}$ \\
J 1846-0258 & $19.340994108^{16}$ & $-4.21955^{16}$ & $24.3^{16}$ & $2.64^{18}$ \\
J 1119-6127 & $15.401361301^{17}$ & $-1.517708^{17}$ & $4.014^{17}$ & $2.684^{18}$ \\
J 1734-3333 & $5.37327178^{19}$ & $-0.104742^{19}$ & $0.018^{19}$ & $0.9^{18}$ \\
\hline
\end{tabular}

Table 2. Time variation of the pulsar's radius, $\dot{R}$.

\begin{tabular}{cc}
\hline Pulsar & $\begin{array}{c}\dot{R} \\
\left(\mathrm{~cm} \mathrm{~s}^{-1}\right)\end{array}$ \\
\hline B $0531+21$ & $1.14 \times 10^{-6}$ \\
B 0833-45 & $5.1 \times 10^{-7}$ \\
B 0540-69 & $1.30 \times 10^{-6}$ \\
B $1509-58$ & $2.809 \times 10^{-7}$ \\
J $1846-0258$ & $1.39 \times 10^{-6}$ \\
J $1119-6127$ & $5.479 \times 10^{-7}$ \\
J $1734-3333$ & $1 \times 10^{-6}$ \\
\hline
\end{tabular}




\section{Conclusions}

By assuming that the radii of pulsars steadily change in time we calculated this rate of change $(\dot{R})$ for pulsars with known braking indices. These values are small and at the present they are not possible to be verified observationally. We speculate that physical mechanisms occurring in the star interior could be responsible for such increase in radius, perhaps similar to a mechanism reported in the literature that deals with the possibility of increasing the moment of inertia from of phase transitions in hybrid stars ${ }^{20}$.

\section{Acknowledgments}

H. O. O. thanks Coordenação de Aperfeiçoamento de Pessoal de Nível Superior (CAPES) for the financial support. N. S. M. acknowledges the National Institute of Science and Technology in Astrophysics (INCT-A, Brazil; a joint CNPq and FAPESP project, FAPESP grant \# 2008/57807-5) for support. The authors acknowledge FAPESP for support under the thematic project \# 2013/26258-4.

\section{References}

1. A. Hewish, J. S. Bell, P. D. H. Pilkington, P. F. Scott and R. A. Collins, Nature 217, 5130 (1968).

2. T. Gold, Nature 218, 731 (1968).

3. F. Pacini, Nature 216, 567 (1967).

4. J. P. Ostriker and J. E. Gunn 1969, ApJ 157, 1395 (1969).

5. P. Goldreich, ApJ 160, L11 (1970).

6. W. W. Macy Jr, ApJ 190, 153 (1974).

7. S. Hinata and E. Jackson, ApJ 192, 703 (1974).

8. N. S. Magalhaes, T. A. Miranda, and C. Frajuca, ApJ, 755, 54 (2012).

9. N. S. Magalhaes, A. S. Okada, and C. Frajuca, MNRAS, 461, 3993 (2016)

10. M. P. Allen and J. E. Horvath, MNRAS, 287, 615 (1997)

11. O. Hamil, astro ph, arXiv:1503.09110 (2015).

12. S. L. Shapiro and S. A. Teukolsky, 2008, Black Holes, White Dwarfs and Neutron Neutron Stars: the physics of compact objects, (John Wiley \& Sons, Hoboken, New Jersey, 2008).

13. A. Lyne, R. Pritchard, and F. Graham Smith, MNRAS 265, 1003 (1993).

14. A. Lyne, R. Pritchard, and F. Graham Smith et al., Nature 381 (1996).

15. P. T. Boyd, G. W. van Citters, and J. F. Dolan et al., ApJ 448, 365 (1995).

16. M. A. Livingstone, V. M. Kaspi, and F. P. Gavriil et al. ApES SS 308, 317 (2007).

17. P. Weltevrede, S. Johnston, and C. M. Espinoza, MNRAS 411, 1917 (2011).

18. R. N. Manchester, G. B. Hobbs, and A. Teoh et al., ApJ 129, 1993 (2005). ATNF Pulsar Catalogue webpage: www.atnf.csiro.au. Access in 09/19/2016.

19. C. M. Espinoza, A. G. Lyne, and M. Kramer et al. ApJ 741, L13 (2011).

20. B. Franzon, R. O. Gomes, and S. Schramm, MNRAS 463, 571 (2016). 